\title{
Inhibitory Effect of Cilnidipine on Pressor Response to Acute Cold Stress in Spontaneously Hypertensive Rats
}

\author{
Masahiro Hosono ${ }^{1}$, Tohru Hiruma ${ }^{1}$, Kiyoshi Watanabe ${ }^{1}$, Yutaka Hayashi ${ }^{1}$, Haruo Ohnishi ${ }^{1}$, \\ Yoshinobu Takata ${ }^{2}$ and Hitoshi Kato ${ }^{2}$ \\ ${ }^{1}$ Pharmaceuticals Research Laboratories, Fujirebio Inc., 51 Komiya-cho, Hachioji, Tokyo 192, Japan \\ ${ }^{2}$ Department of Pharmacology, Faculty of Pharmaceutical Sciences, Teikyo University, Sagamiko, Kanagawa 199-01, Japan
}

Received May 16, 1995 Accepted July 18, 1995

\begin{abstract}
We investigated the effect of cilnidipine on cardiovascular and neuroendocrine responses to acute cold stress in conscious and unrestrained or moderately restrained spontaneously hypertensive rats (SHRs). Acute cold stress significantly increased mean blood pressure without marked change in heart rate. The acute cold stress-induced elevation in blood pressure was almost abolished by $1 \mathrm{mg} / \mathrm{kg}, \mathrm{p} . \mathrm{o}$. of prazosin. The cold stress also elevated plasma norepinephrine and epinephrine levels. Cilnidipine at $3 \mathrm{mg} / \mathrm{kg}$, p.o. significantly inhibited the pressor response to acute cold stress. Although $3 \mathrm{mg} / \mathrm{kg}$, p.o. of nifedipine, nicardipine or manidipine lowered mean blood pressure to a similar extent as cilnidipine, none of these three drugs affected the pressor response. Cilnidipine also reduced the cold stress-induced increment in plasma norepinephrine but not the epinephrine level. These findings suggest that acute cold stress may induce the elevation in blood pressure due to an enhanced activation of the sympathoadrenal system in SHRs and that cilnidipine may suppress the pressor response by inhibiting the sympathetic nerve activity.
\end{abstract}

Keywords: Cilnidipine, Acute cold stress, Pressor response, Plasma catecholamine level, Spontaneously hypertensive rat

It is well-known that stress is one of the risk factors of cardiovascular diseases such as hypertension. Stress is considered to play an important role in the development and deterioration of hypertension (1-3). In clinical studies, many investigators have reported cardiovascular and neuroendocrine responses to stress using various procedures such as the cold pressor test, arithmetic test and hand grip test. These reports have shown that the stress increased both the blood pressure and plasma catecholamine levels and that the response to stress was enhanced in hypertensive subjects (1). Sudden changes in blood pressure by stress might be related to the incidence of hypertensive complications (4). Thus, it is important to investigate the effect of antihypertensive drugs on cardiovascular responses to stress. Dihydropyridine (DHP) calcium (Ca) channel blockers have been used as antihypertensives. These drugs are roughly classified into two groups. One is the conventional type, such as nifedipine and nicardipine, that lowers blood pressure rapidly. Another is a recently developed type that has a slow-onset and long-lasting hypotensive action, such as manidipine (5). It is of interest to analyze whether Ca channel block- ers can affect cardiovascular responses to stress.

Cilnidipine is a DHP Ca channel blocker synthesized at Fujirebio, Inc. It has been shown that cilnidipine inhibits both depolarization-induced ${ }^{45} \mathrm{Ca}^{2+}$ influx in cultured rat vascular smooth muscle cells (6) and $\mathrm{Ba}^{2+}$ inward current in rabbit basilar artery (7). Cilnidipine has a slow-onset and long lasting antihypertensive action in SHRs (8), which is based on its Ca channel blocking action (9). In the present study, we investigated the effect of cilnidipine on cardiovascular and neuroendocrine responses to acute cold stress in conscious spontaneously hypertensive rats (SHRs).

\section{MATERIALS AND METHODS}

\section{Animals used}

Male 15-week-old SHRs (Charles River Japan, Yokohama) weighing $320-440 \mathrm{~g}$ were used. The animals were housed in individual cages under identical conditions of temperature $\left(22 \pm 2^{\circ} \mathrm{C}\right)$, humidity $(55 \pm 15 \%)$ and a $12-\mathrm{hr}$ light/dark cycle, and they were allowed to have normal rat chow and tap water ad libitum. 
Blood pressure measurement by means of the telemetry system

For the blood pressure measurement, we used a telemetry system (Data Quest 4; Data Sciences, Inc., St. Paul, MN, USA) consisting of an implantable radio-telemetry device (transmitter), a receiver and a computerbased data acquisition system. The transmitter contains a pressure transducer, a battery and a radio-frequency transmitter. This system is capable of continuous monitoring and recording arterial blood pressure and heart rate under the conscious and unrestrained condition. SHRs were anesthetized with sodium pentobarbital (Nembutal ${ }^{\mathbb{R}}$; Abbott Laboratories, North Chicago, IL, USA) at $50 \mathrm{mg} / \mathrm{kg}$, i.p., and the abdomen was opened by midline incision. The descending aorta was exposed, and the catheter of the transmitter (TA11PA-CA; Data Sciences, Inc.) was retrogradely inserted $1.0 \mathrm{~cm}$ into the abdominal aorta below the renal artery. Then the catheter was secured with medical grade tissue adhesive and a small cellulose fiber patch to promote fibrotic tissue formation. The body of the transmitter was affixed to the inner peritoneal wall. Sterile technique was employed throughout the transmitter implantation procedure. Experiments were started 2 weeks after the surgery.

\section{Experiments on cardiovascular response to cold stress}

As shown in Fig. 1, SHRs were each placed in an individual cage, and blood pressure and heart rate of conscious and freely moving SHRs were continuously measured by means of the telemetry system. The individual cage wherein a rat had been placed was gently lowered and sunk 2-cm-deep into ice-cold water at $4^{\circ} \mathrm{C}$ for $1 \mathrm{~min}$. These procedures allowed the rat to sink into the ice-cold water up to all four paws. Thus, SHRs were exposed to acute cold stress. The cold stress loading was intermittently repeated 7 times every $30 \mathrm{~min}$. This repetitive cold stress loading procedure was repeated in each animal 4 times at 3-day intervals, which were designated as Days 1-4. On Day 4, prazosin was administered orally $1 \mathrm{~min}$ after the first cold stress loading. One week was allowed for the washout period of prazosin effects. Thereafter, the stress procedure, which consisted of 7 loadings a day, was further repeated in each animal once every week. During this period, each $\mathrm{Ca}$ channel blocker was given $1 \mathrm{~min}$ after the first stress loading of each day.

\section{Experiments on neuroendocrine response to cold stress}

Arterial and venous catheters were implanted into SHRs under anesthesia with sodium pentobarbital at 50 $\mathrm{mg} / \mathrm{kg}$, i.p. on the day before the experiment. The arterial catheter was inserted into right carotid artery for the collection of blood samples. The venous catheter was inserted into left external jugular vein for injection of a blood substitute. The other end of the catheters was exteriorized at the back of the neck through the skin and fixed there. Each catheter was filled with heparin (Novo Nordisk, Bagsvaerd, Denmark), 1000 units/ml in saline. On the following day, SHRs were placed in a specially designed holder that provided moderate restraint of the limbs while allowing free movement of the head. A $600-\mu \mathrm{l}$ aliquot of arterial blood was drawn via the catheter by a syringe containing disodium ethylenediaminetetraacetic acid (Dojin Co., Tokyo) within $30 \mathrm{sec}$. The blood sample was immediately centrifuged at $3000 \mathrm{rpm}$ for $10 \mathrm{~min}$ at $4^{\circ} \mathrm{C}$, and plasma was removed and stored at $-80^{\circ} \mathrm{C}$ until used for the determinations of norepinephrine (NE) and

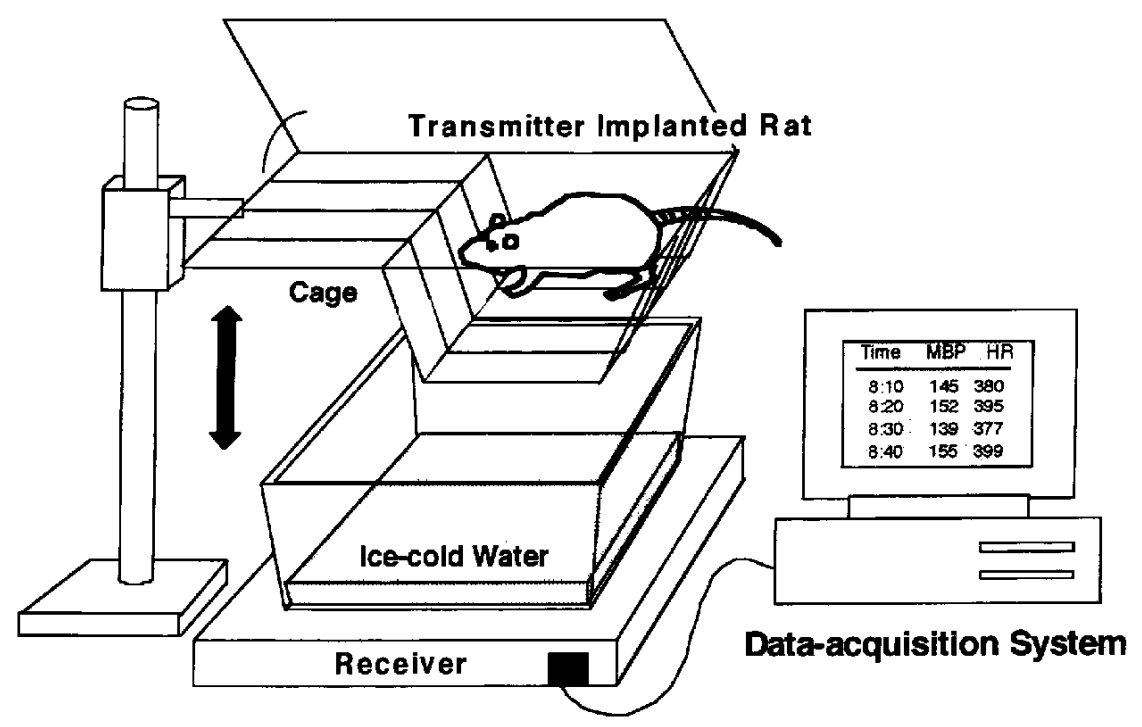

Fig. 1. Schematic diagram of experimental setup for measurement of cardiovascular response to acute cold stress. 


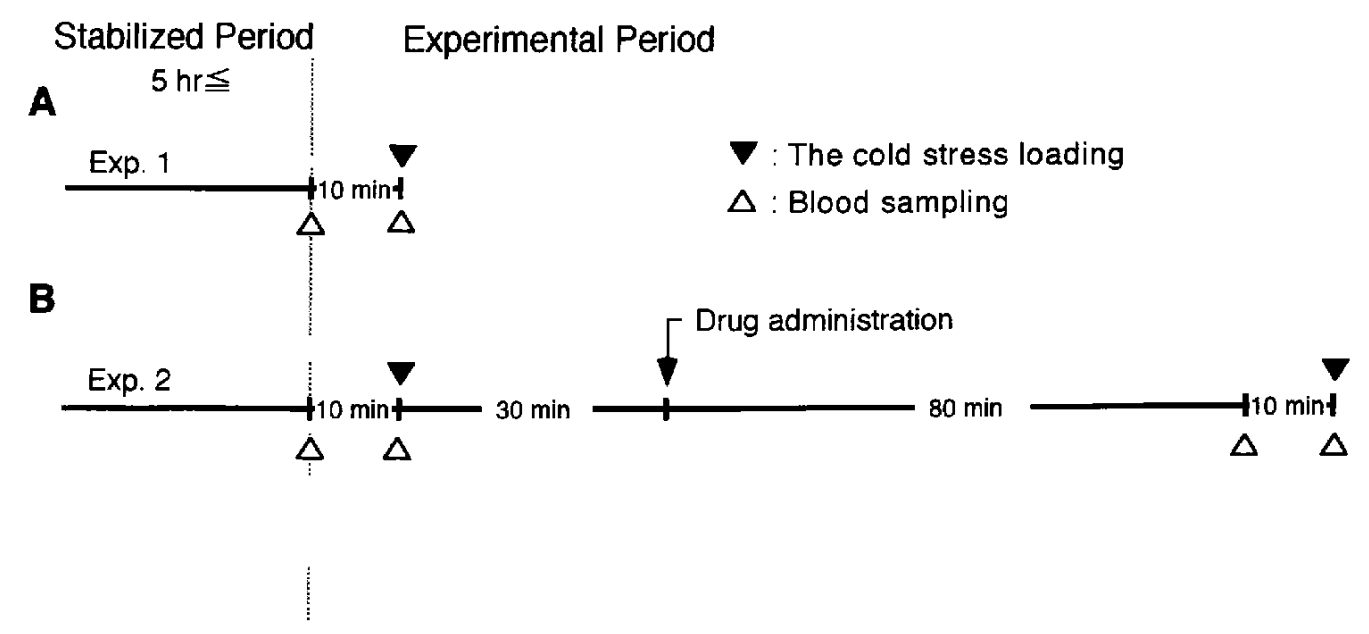

Fig. 2. Experimental protocol for determination of neuroendocrine response to acute cold stress.

epinephrine (E). To compensate for fluid losses due to blood sampling, $600 \mu \mathrm{l} 0.5 \%$ bovine serum albumin in saline at $37^{\circ} \mathrm{C}$ was infused through the venous catheter after each blood sampling.

\section{Experimental protocol for plasma $N E$ and $E$ measure-} ment

Effect of acute cold stress on plasma $N E$ and $E$ levels: In experiments in which the effect of acute cold stress on plasma NE and E levels was studied, as shown in Fig. 2A, moderately restrained SHRs were exposed to acute cold stress only once after a stabilization period of $5 \mathrm{hr}$ or more. Blood sampling was performed $10 \mathrm{~min}$ before acute cold stress and during the last $30 \mathrm{sec}$ of the acute cold stress period.

Effect of cilnidipine on acute cold stress-induced increment in plasma $N E$ and $E$ levels: In this series of experiments, as shown in Fig. 2B, acute cold stress loading was performed twice at 2 -hr intervals after a stabilization period. A blood sample was collected $10 \mathrm{~min}$ before each cold stress and during the last $30 \mathrm{sec}$ of each cold stress period. Cilnidipine $(3 \mathrm{mg} / \mathrm{kg}$ ) or vehicle was administered orally $90 \mathrm{~min}$ before the second cold stress loading. Prior to the assay, aliquots of plasma $(150 \mu \mathrm{l}) \mathrm{kept}$ at $-80^{\circ} \mathrm{C}$ were mixed with $300 \mu \mathrm{l} 5 \%$ perchloric acid containing $0.6 \%$ sodium acetate, and the mixture was centrifuged at $2000 \mathrm{rpm}$ for $20 \mathrm{~min}$ at $4{ }^{\circ} \mathrm{C}$. Thus, a protein-free supernatant was obtained. NE and $E$ concentrations in the supernatant were measured by means of an automatic catecholamine analyzer (HLC725CA; Toso Co., Tokyo) based on the diphenyl ethylenediamine method. The increment rate of plasma $\mathrm{NE}$ and $\mathrm{E}$ levels by acute cold stress in the presence or absence of cilnidipine was calculated by means of the following formula described by Dahlöf (10):

$$
\begin{aligned}
& \Delta \% \text { in NE (or E) }(\%) \\
& \quad=\left(\frac{\text { plasma NE (or E) during stress loading }}{\text { plasma NE (or E) before stress loading }}-1\right) \times 100 \\
& \text { Increment Rate (\%) } \\
& \quad=\frac{\Delta \% \text { in NE (or E) after drug administration }}{\Delta \% \text { in NE (or E) before drug administration }} \times 100
\end{aligned}
$$

\section{Drugs}

Cilnidipine (Fujirebio Inc., Tokyo), nifedipine and nicardipine hydrochloride (Sigma Chemical Co., St. Louis, MO, USA), manidipine hydrochloride (Calslot ${ }^{\circledR}$; Takeda Chemical Ind., Osaka) and prazosin hydrochloride (Tokyo Kasei Kogyo Co., Tokyo) were suspended in $5 \%$ arabic gum solution. All drugs were administered orally in a volume of $5 \mathrm{ml} / \mathrm{kg}$.

\section{Statistical analyses}

Results are expressed as the mean \pm S.E. Statistical analyses were carried out with the paired Student's $t$-test within one group and the unpaired $t$-test between two groups. Significant differences among three or more groups were determined by one-way analysis of variance followed by Dunnett's multiple comparison test. In each case, $P$ values less than 0.05 were considered statistically significant.

\section{RESULTS}

Cardiovascular response to acute cold stress in conscious and unrestrained SHR

Mean blood pressure and heart rate responses to acute cold stress in conscious and unrestrained SHRs are shown in Fig. 3. On Day 1, the first cold stress loading significantly increased the blood pressure by $32 \pm 4 \mathrm{mmHg}$ $(n=6)$. The elevated blood pressure was returned rapidly to the baseline value after the termination of stress load- 


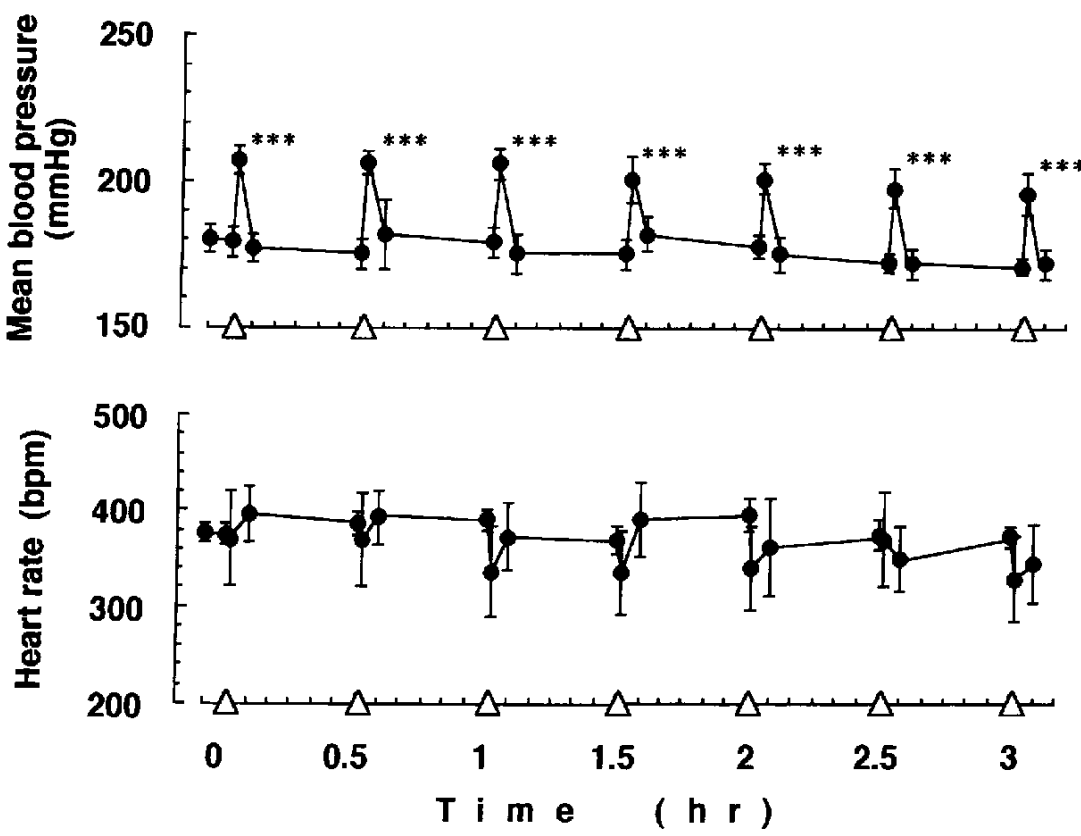

Fig. 3. Changes in mean blood pressure and heart rate by repetitive acute cold stress loadings in conscious and unrestrained SHRs. Stress was given 7 times at 30-min intervals, which was indicated by open triangles. Each value represents the mean \pm S.E. of 6 animals. ${ }^{* * *} \mathrm{P}<0.001$, significantly different from each pre-stress value.
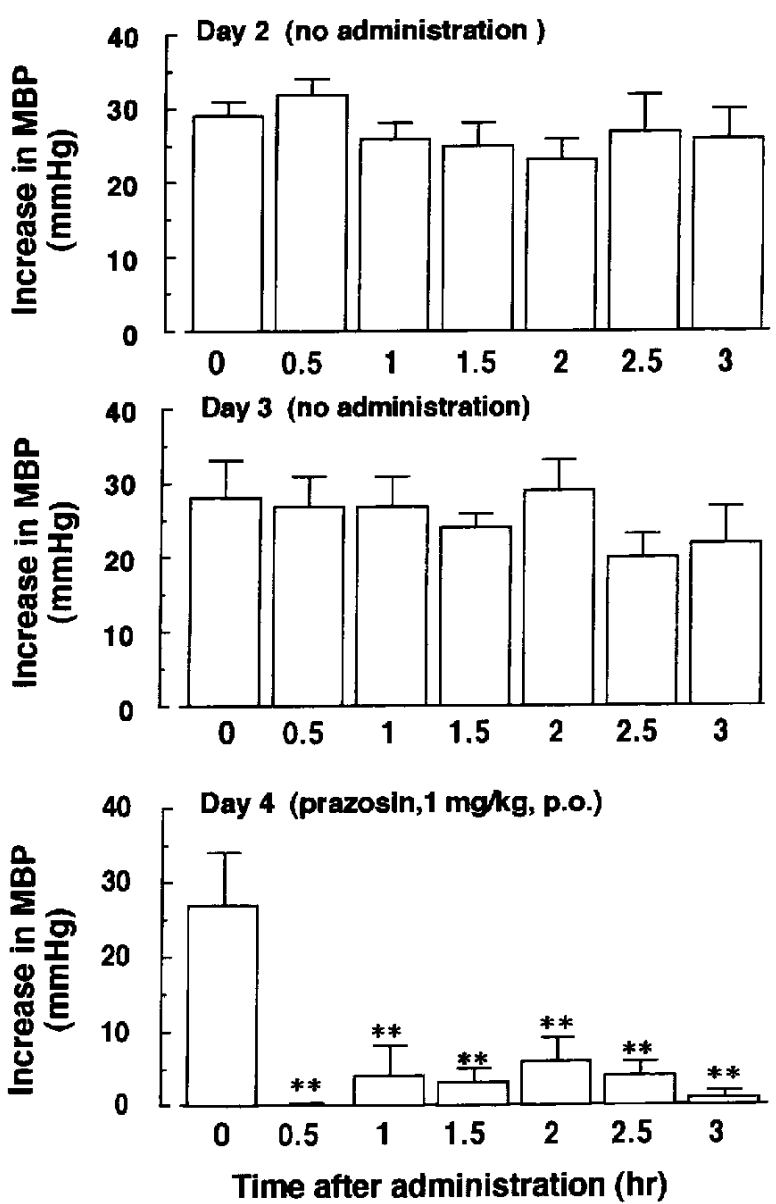

ing. When acute cold stress loadings were repeated 7 times every $30 \mathrm{~min}$, there was no significant difference among these pressor responses. In contrast, the heart rate was not significantly changed by each stress loading. The same repetitive stress loading procedure was repeated in each animal 4 times at 3-day intervals, and the reproducible pressor responses to cold stress were observed on not only Day 1 but also Days 2 and 3 (Fig. 4). On Day 4, the effect of prazosin at $1 \mathrm{mg} / \mathrm{kg}$, p.o. on acute cold stressinduced elevation in blood pressure was determined. Prazosin almost abolished the elevated blood pressure in response to cold stress (Fig. 4).

Effects of Ca channel blockers on the pressor response to the cold stress in conscious and unrestrained SHR

The effects of four Ca channel blockers on the pressor response to cold stress are shown in Fig. 5. Cilnidipine at $3 \mathrm{mg} / \mathrm{kg}$, p.o. reduced the pressor response, which was

Fig. 4. Effect of prazosin at $1 \mathrm{mg} / \mathrm{kg}, \mathrm{p} .0$. on the acute cold stressinduced elevation in mean blood pressure (MBP) in conscious and unrestrained SHRs. Stress was given 7 times at 30 -min intervals on each day. This repetitive cold stress loading procedure was repeated 4 times at 3-day intervals and designated as Days 1-4. Upper, middle and lower graphs show the results on Days 2, 3 and 4, respectively. On Day 4, prazosin was administered 1 min after the first stress loading (time 0). Each value represents the mean \pm S.E. of 6 animals. ${ }^{* *} \mathrm{P}<0.01$, significantly different from the value at time 0 on Day 4. 

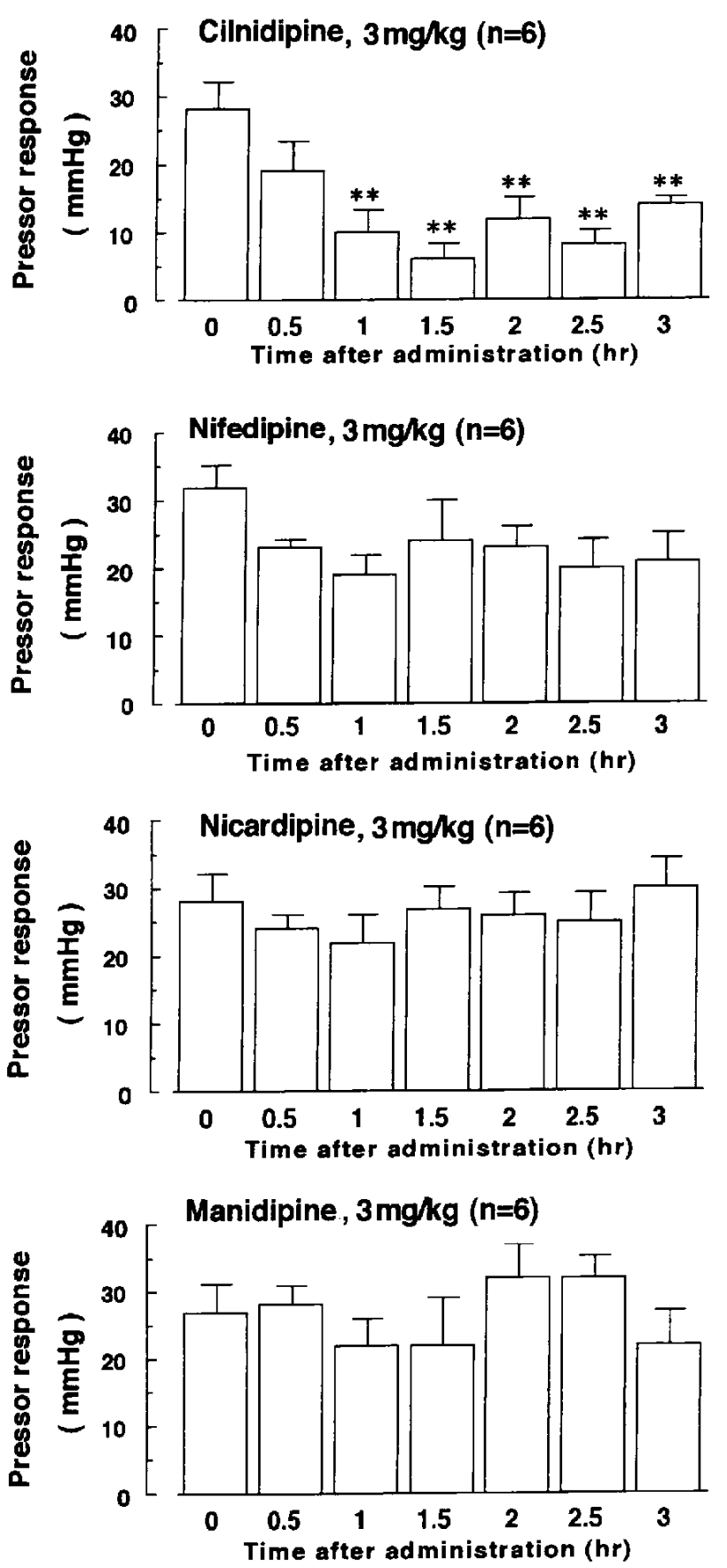

Fig. 5. Effects of cilnidipine, nifedipine, nicardipine and manidipine, each at $3 \mathrm{mg} / \mathrm{kg}$, p.o., on the pressor response to acute cold stress in conscious and unrestrained SHRs. Each value represents the mean \pm S.E. of 6 animals. ${ }^{* *} \mathrm{P}<0.01$, significantly different from the value at time 0 .

significant $1 \mathrm{hr}$ and reached its peak effect $1.5 \mathrm{hr}$ after administration. In addition, this reduction was sustained for at least $3 \mathrm{hr}$. As shown in Fig. 6, cilnidipine at doses of 1 and $3 \mathrm{mg} / \mathrm{kg}$, p.o. caused a dose-dependent inhibition of the pressor effect of cold stress. Cilnidipine at 3 $\mathrm{mg} / \mathrm{kg}$, p.o. inhibited the pressor response by $78 \pm 6 \% 1.5$

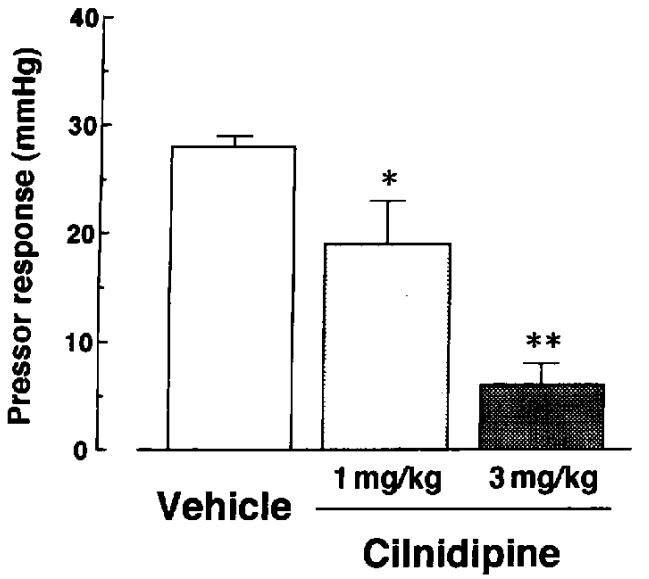

Fig. 6. Effects of cilnidipine at 1 and $3 \mathrm{mg} / \mathrm{kg}, \mathrm{p} .0$. on the pressor response to acute cold stress in conscious and unrestrained SHRs. Cilnidipine effects at $1.5 \mathrm{hr}$ after administration are shown. Each value represents the mean \pm S.E. of 6 animals. ${ }^{*} \mathrm{P}<0.05$ and ${ }^{* *} \mathrm{P}<0.01$, significantly different from the vehicle group.

$\mathrm{hr}$ after administration. On the other hand, nifedipine, nicardipine and manidipine at each $3 \mathrm{mg} / \mathrm{kg}$, p.o. elicited no significant effects on acute cold stress-induced elevation in blood pressure (Fig. 5).

Hypotensive action of Ca channel blockers in conscious and unrestrained SHR

Time courses of the changes in mean blood pressure of SHRs in the repetitive cold stress loading experiments are shown in Fig. 7. Basal values of mean blood pressure in

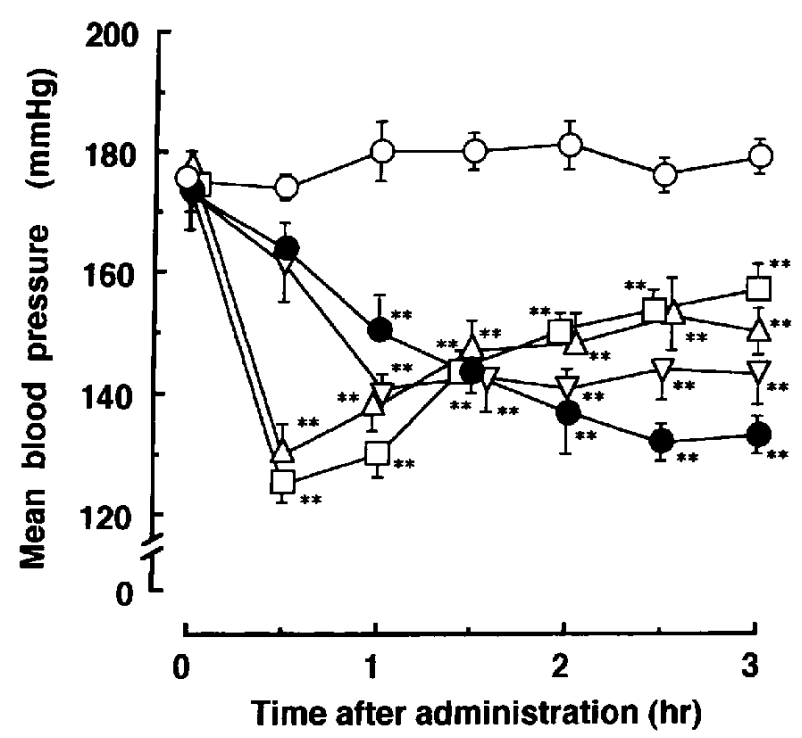

Fig. 7. Effects of cilnidipine (O), nifedipine $(\triangle)$, nicardipine $(\square)$ and manidipine $(\nabla)$ at each $3 \mathrm{mg} / \mathrm{kg}$, p.o. or vehicle $(\bigcirc)$ on mean blood pressure in conscious and unrestrained SHRs. Each point represents the mean \pm S.E. of 6 animals. ${ }^{* *} \mathrm{P}<0.01$, significantly different from the corresponding vehicle value. 


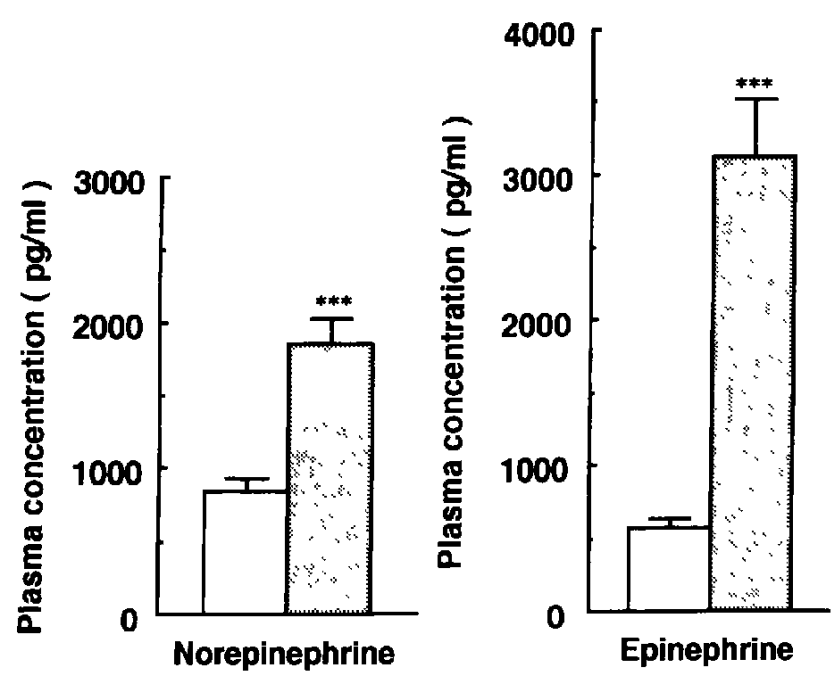

Fig. 8. Plasma norepinephrine and epinephrine levels before (open column) and during (hatched column) acute cold stress loading in conscious SHRs. Each value represents the mean \pm S.E. of 10 animals. ${ }^{* * *} \mathrm{P}<0.001$, significantly different from the value before stress loading.

the vehicle, cilnidipine, nifedipine, nicardipine and manidipine groups were $176 \pm 4,173 \pm 6,178 \pm 2,174 \pm 2$ and $173 \pm 4 \mathrm{mmHg}(\mathrm{n}=6)$, respectively. There was no significant difference among these basal values. Each drug at $3 \mathrm{mg} / \mathrm{kg}$, p.o. lowered the mean blood pressure. The maximum hypotensive effects of cilnidipine, nifedipine, nicardipine and manidipine were obtained at 2.5, 0.5, 0.5 and $2 \mathrm{hr}$ following administration, respectively. At these corresponding times, cilnidipine, nifedipine, nicardipine and manidipine reduced the mean blood pressure by $42 \pm 5,49 \pm 5,49 \pm 2$ and $34 \pm 3 \mathrm{mmHg}(\mathrm{n}=6)$, respectively. There was no significant difference among the maximum hypotensive effects of the four blockers.
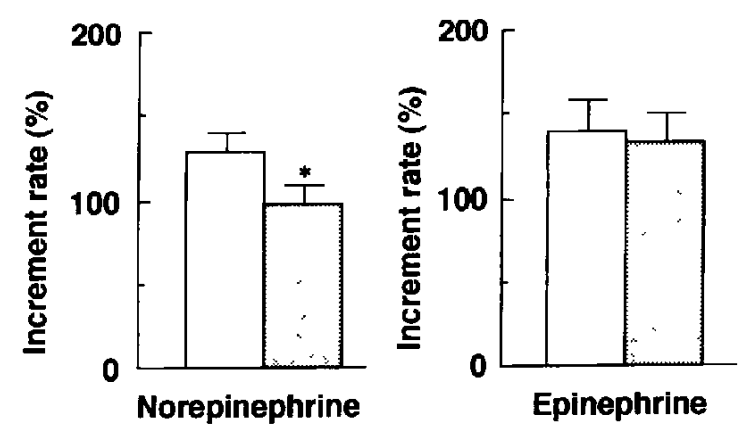

Fig. 9. Effect of cilnidipine on the increment in plasma norepinephrine and epinephrine levels in response to acute cold stress in conscious SHRs. Plasma norepinephrine and epinephrine levels were determined using a blood sample obtained at $1.5 \mathrm{hr}$ after vehicle (open column) and $3 \mathrm{mg} / \mathrm{kg}$, p.o. cilnidipine (hatched column) administration. Each value represents the mean \pm S.E. of 10 animals. ${ }^{*} \mathrm{P}<0.05$, significantly different from the vehicle group.
The cold stress-induced changes in plasma $N E$ and $E$ levels

Figure 8 shows plasma NE and $\mathrm{E}$ levels before and during cold stress in conscious and moderately restrained SHRs. The cold stress significantly elevated plasma NE and $\mathrm{E}$ levels. Plasma NE and $\mathrm{E}$ levels during cold stress were approx. twofold and fivehold higher than those before cold stress, respectively.

Effect of cilnidipine on acute cold stress-induced increment in plasma $N E$ and $E$ levels

As shown in Fig. 9, cilnidipine at $3 \mathrm{mg} / \mathrm{kg}$, p.o. suppressed the acute cold stress-induced increment in plasma NE level by about $25 \%$, but it failed to affect the increment in plasma $E$ level induced by acute cold stress.

\section{DISCUSSION}

It has been already reported in both clinical and preclinical studies that various kinds of stressors may increase blood pressure and/or heart rate due to activation of the sympathetic nervous system (1). Acute cold stress is one of these stressors. There are many reports regarding the response to acute cold stress of hypertensive patients but not of SHRs. In the present study, we used the telemetry system for continuous measurements of blood pressure and heart rate in conscious and unrestrained SHRs and were able to demonstrate the blood pressure elevation produced by acute ice-cold stress loading, as described previously in human subjects. The pressor effect in response to cold stress was almost abolished by prazosin. Acute cold stress showed no significant effects on heart rate in SHRs. Thus, it appears that the pressor response to acute cold stress in SHRs may probably be due to enhanced vasoconstriction via $\alpha_{1}$-adrenergic receptors rather than increased cardiac output. We also demonstrated that acute cold stress remarkably increased the plasma NE level in conscious SHRs. In clinical study, a similar result was described by LeBlanc et al. (11). It was reported that changes in plasma NE level correlate with the activity of sympathetic nervous system in both rats $(12,13)$ and human subjects (14). Therefore, it is thought that acute cold stress activates the sympathetic nervous system in SHRs, resulting in vasoconstriction.

The pressor effect of cold stress in SHRs was reproducible, which seems to be suitable for evaluation of drug effects. So, we also evaluated the effects of some $\mathrm{Ca}$ channel blockers on the pressor response to cold stress. Cilnidipine at 1 and $3 \mathrm{mg} / \mathrm{kg}$, p.o. inhibited the pressor response significantly and dose-dependently. In contrast, nifedipine, nicardipine and manidipine at $3 \mathrm{mg} / \mathrm{kg}$, p.o. did not significantly affect it. The three blockers lowered blood pressure to a similar extent as $3 \mathrm{mg} / \mathrm{kg}$ of cilnidi- 
pine. Moreover, with respect to the onset-time or duration of hypotensive action, cilnidipine was almost the same as manidipine. These results suggest that the hypotensive action of cilnidipine based on $\mathrm{Ca}$ channel blocking action may not contribute to the inhibition of the pressor response to cold stress.

It has been reported that cilnidipine failed to inhibit the vasocontraction induced by exogenous NE in the rabbit aorta (9), suggesting that the blocker has no $\alpha$-adrenergic blocking action. Therefore, it is unlikely that the inhibitory effect of cilnidipine on the pressor response may be due to a postsynaptic $\alpha$-adrenoceptor blocking action.

In this study, we further investigated the effect of cilnidipine on the sympathoadrenal system by measuring plasma NE and E levels. Cilnidipine significantly suppressed the elevation in plasma NE level in response to cold stress in SHRs, whereas it had no effects on the acute cold stress-induced increase in plasma $\mathrm{E}$ level. Thus, cilnidipine may inhibit the pressor response to cold stress by reducing the sympathetic nerve activity without affecting the adrenal medulla.

Although the exact mechanisms by which cilnidipine inhibits the sympathetic nerve activity remain unclear, the following four sites of action should be considered: 1) suppression of the reflex via the central nervous system (CNS), 2) ganglion blocking, 3) anesthesia on neurons and 4) inhibition of neurotransmission in nerve endings. Since Watanabe et al. (15) have reported that cilnidipine had neither any action on the CNS or ganglion nor a local anesthetic action in its general pharmacological studies, the former three candidates seem to be unrelated to the sympatho-inhibitory effect of cilnidipine. Rather, the last of them could be related to the sympatho-inhibitory effect of cilnidipine. We are currently performing studies to obtain direct evidence for the inhibitory effect of cilnidipine on NE release from the sympathetic nerve endings.

In conclusion, these results suggest that acute cold stress may induce the pressor response due to an enhanced activation of the sympathoadrenal system in SHRs and that cilnidipine may suppress the pressor response by inhibiting the sympathetic nerve activity.

\section{Acknowledgments \\ We wish to thank Miss Masako Yatabe for her technical assist- ance.}

\section{REFERENCES}

1 Herd JA: Cardiovascular response to stress. Physiol Rev 71, $305-330$ (1991)
2 Galosy RA, Clarke LK, Vasko MR and Crawford IL: Neurophysiology and neuropharmacology of cardiovascular regulation and stress. Neurosci Biobehav Rev 5, 137-175 (1981)

3 Widgren BR, Wikstrand J, Bergland $G$ and Andersson $O K$ : Increased response to physical and mental stress in men with hypertension parents. Hypertension 20, 606-611 (1992)

4 Gelernt MD and Hochman JS: Acute myocardial infarction triggered by emotional stress. Am J Cardiol 69, 1512-1513 (1992)

5 Kakihana M, Suno M and Nagaoka A: Antihypertensive effect of $\mathrm{CV}-40932 \mathrm{HCl}$, a new calcium antagonist, in three rat models of hypertension. Jpn J Pharmacol 48, 223-228 (1988)

6 Yoshimoto R, Dohmoto H, Yamada K and Goto A: Prolonged inhibition of vascular contraction and calcium influx by the novel 1,4-dihydropyridine calcium antagonist cinaldipine (FRC-8653). Jpn J Pharmacol 56, 225-229 (1991)

7 Oike $\mathrm{M}$, Inoue $\mathrm{Y}$, Kitamura $\mathrm{K}$ and Kuriyama $\mathrm{H}$ : Dual action of FRC8653, a novel dihydropyridine derivative, on the $\mathrm{Ba}^{2}$. current recorded from the rabbit basilar artery. Circ Res 67, $993-1006(1990)$

8 Ikeda $\mathrm{K}$, Hosono $\mathrm{M}$, Iida $\mathrm{H}$ and Ohnishi $\mathrm{H}$ : Antihypertensive and cardiovascular profiles of a newly sythesized dihydropyridine derivative 2-methoxyethyl (E)-3-phenyl-2-propen-1yl( \pm )-1,4-dihydro-2,6-dimethyl-4-(3-nitrophenyl) pyridine-3,5dicarboxylate (FRC-8653). Pharmacometrics 44, 433-442 (1992)

9 Hosono $\mathrm{M}$, Iida $\mathrm{H}$, Ikeda $\mathrm{K}$, Hayashi $\mathrm{Y}$, Dohmoto $\mathrm{H}$, Hashiguchi $\mathrm{Y}$, Yamamoto $\mathrm{H}$, Watanabe $\mathrm{N}$ and Yoshimoto $\mathrm{R}$ : In vivo and ex vivo $\mathrm{Ca}$-antagonistic effect of 2-methoxyethyl (E)-3-phenyl-2-propen-1-yl( \pm )-1,4-dihydro-2,6-dimethyl-4(3-nitrophenyl) pyridine-3,5-dicarboxylate (FRC-8653), a new dihydropyridine derivative. J Pharmacobiodyn 15, 547-553 (1992)

10 Dahlöf P: Modulatory interactions of neuropeptide Y (NPY) on sympathetic neurotransmission. Acta Physiol Scand 586, $1-85$ (1989)

11 LeBlanc J, Cote J, Jobin M and Labrie A: Plasma catecholamines and cardiovascular responses to cold and mental activity. J Appl Physiol 47, 1207-1211 (1979)

12 Castagne V, Corder R, Gaillard R and Mormede P: Stressinduced changes of circulating neuropeptide $Y$ in the rat: comparison with catecholamines. Regul Pept 19, 55-63 (1987)

13 Dahlöf $\mathrm{C}$ and Dahlöf $\mathrm{P}$ : Adrenaline (A) as a physiological agonist for presynaptic $\beta$-adrenoceptors mediating facilitation of noradrenaline (NA) release. Abstract of the 9th IUPHAR, London, p 341 (1984)

14 Wallin BG: Muscle sympathetic activity and plasma concentration of noradrenaline. Acta Physiol Scand 527, 21-24 (1984)

15 Watanabe K, Dozen M, Maeda E, Hosono M, Kase N, Ikeda K, Iida $H$ and Hayashi $Y$; General pharmacological studies of 2methoxyethyl (E)-3-phenyl-2-propen-1-yl( \pm )-1,4-dihydro-2,6dimethyl-4-(3-nitrophenyl) pyridine-3,5-dicarboxylate (FRC8653), a new antihypertensive drug. Pharmacometrics 44, $443-463$ (1992) (Abstr in English) 